The most common form of disease of the vertebræ is the strumous, with angular curvature, and often with lumbar or psoas abscess. The bones of the spine, too, are sometimes the seat of malignant disease. The spinal cord and its membranes may become secondarily implicated in cases of primary disease or injury of their bony sheath, but more frequently disease of the cord and its coverings is unconnected with disease of the vertebræ. In cases of spinal meningitis, there is, as a rule, more pain in the back than when the substance of the cord is alone affected. The pain is usually increased by movement, by percussion on the spine over the seat of disease, and by hot applications to the surface. The pain is often referred to the extremities of the nerves which pass out from the part affected, and is commonly associated with numbness, a sense of tingling, and other perverted sensations.

A sense of constriction across the chest is a frequent symptom. There is often spasmodic twitching of the muscles; and in proportion to the affection of the substance of the cord is the frequency of paralysis (paraplegia) affecting the parts below the seat of disease, and implicating not only the voluntary muscles, but also those of the bladder and rectum, with resulting retention or incontinence of urine and fæces.

Disease of the spinal cord itself is usually attended with less pain than when the membranes are the seat of disease. Inflammation of the substance of the cord (myelitis) may quickly result in paraplegia, with little or no local pain in the back. In some cases of spinal hamorrhage, too, paralysis below the seat of pressure has been associated with little or no pain; while, in others, more or less severe dorsal pain has marked the onset of the hæmorrhage.

Pressure on the spinal cord by a tumour, fibrous, strumous, or cancerous; has often been associated with severe pain in the back. In a case recorded by Mr. Shaw (Pathological Transactions, vol. ii, p. 24), paraplegia was caused by two scrofulous tumours occupying the interior and lower part of the spinal cord, and invested on all sides by a thin layer of medullary matter. In that case, the pain in the lumbar region was so severe that the symptoms were supposed to result from caries of the vertebræ; and the more so, as there was a slight projection of one of the lumbar spinous processes.

The pain resulting from a tumour pressing on the spinal cord is very variable. In one case quoted by Dr. Abercrombie, the first symptom was neuralgic pain in the arm, which diminished as paralysis came on; in another case, the patient had sciatic pain extending to the toes. Mostly the pain is referred to the back, and indicates the seat of the disease; thence it radiates in the direction of the nerves whose roots are invaded. When there is no actual pain, there may be modifications of sensation, such as a sense of coldness or heat, or sudden alternations of these; numbness and pricking, or formication. Next in frequency to pain are muscular contractions in the affected limbs, followed in some cases by rigid flexion of a limb, and attended by a great susceptibility to the excito-motor stimulus, and, in a yet further stage, by complete paralysis. In short, the effect of moderate pressure on the cord is to cause spasm and neuralgia, passing on, with increase of the lesion, to paralysis and complete anæsthesia.

I scarcely need warn you to be vigilant and careful not to mistake any of these formidable diseases of the spinal cord and its membranes for the muscular and rheumatic affections to which $I$ referred in the earlier part of this lecture.

There is an acute and transient form of backache which probably has its seat in the spinal cord, I allude to the pain in the back which occurs at the commencement of many acute diseases, especially the febrile exanthemata, and which is usually much complained of during the initiatory fever of small-pox. In some cases which have proved to be nothing more serious than febrile catarrh, the initiatory backache has been so severe as to excite a suspicion of incipient small-pox.

EPILEPSY PRODUCED BY PiCrotoxin.-Drs. Chirone and Testa thus sum up the result of their researches on the action of picrotoxin, in the Annali Universali di Medicina e Chirurgia for April I880. I. Picrotoxin is capable of producing a true artificial epilepsy. 2. This epilepsy is independent of the psycho-motor centres; it is, indeed, more intense after their removal. 3. The picrotoxin acts first on the medulla oblongata and on the commissural fibres between the brain and spinal cord, and then on the spinal centres themselves. 4. This shows the existence of a functional antagonism between the psychomotor centres, and the motor centres of the medulla oblongata and spinal cord. 5. The convulsive movements of the limbs produced by picrotoxin are due in the first place to its action on the medulla oblongata. This action is propagated to the spinal cord. 6. In the frog, the influence on the spinal functions is more marked than on the cerebral; while in higher animals the cerebral motor centres are more affected. 7. Cinchonidin is capable of producing epilepsy of cerebral origin; picrotoxin, epilepsy of spinal origin.

\section{CLINICAL: LECTURE}

\section{SOME VARIETIES OF NERVOUS HEADACHE.}

\author{
Delivered at the Samaritan Hospital.
} By W I I. L I A M H E N R Y D A Y, M.D.,
Physician to the Hospital.

2. Neuralgic Headache.-In my last lecture (BRITISH Medical JourNaL, January 24th, I880), I spoke of nervous headache, and I attempted to divide the subject into four varieties. I alluded to the characters of each variety, and I remarked that it was difficult to assign to each a separate and independent classification. To-day, I will devote myself to the consideration of that variety of nervous headache which is generally unilateral, seizing one side of the head, and is recognised as neuralgic headache. I say generally unilateral, because I have known the paroxysms of frontal headache to recur so regularly, and to last such a definite length of time, and, moreover, to produce such characteristic sensations belonging to the neuralgic type, as to bring them within this category, and to yield to the same remedial treatment.

I shall have to speak, as we proceed, of some curious features in connection with this form of headache, its mode of origin, severity, duration, and locality; how various are the phenomena in different individuals, though fundamentally agreeing in general principles. In support of these facts, I shall cite some examples, giving such an explanation as each case may appear to justify.

Neuralgic headache is a type that deserves our attentive study, since it is as common as it is obstinate to cure.

Just as recurring neuralgia of the sciatic nerve ultimately impairs the sensation of the limb, and weakens its muscular power, so will recurring attacks of neuralgic headache affect the nervous tissue of the brain, till the patient is no longer able to exercise his intellect as he did formerly, or even to undergo the ordinary avocations of life, without feeling a strain on his nerves, which is certain to become worse.

I would define neuralgic headache as that variety of the nervous form in which the trunk or the special branches of a nerve are affected. It is a local pain affecting one side of the head. Unlike frontal headache, it is an unsymmetrical pain; its origin is one-sided. Now, the fifth nerve, either at its origin, or in some part of its course, is oftenest implicated. Its deep origin from the medulla is significant, when we bear in mind that the centre of the vaso-motor system is also traced to the medulla oblongata. The transference of impressions due to peripheral irritation (through cold, decayed teeth, etc.) or to central mischief, from excessive or diminished blood-supply, depending on morbid vaso-motor conditions, or through mental troubles acting on the sensorium, can be readily understood, when this association of brain, medulla, and fifth nerve is remembered. In any particular case of headache, $a$ correct and precise explanation of its pathology is uncertain, owing to the difficulty of tracing the fibres of any one peripheral nerve to its centre, and likewise to the innumerable complications which may engender fallacies in clinical examination.

I would, however, impress this point upon you, as it is worth our serious notice. The causes which invite nervous headache in most instances are the same, or closely allied to those, which originate neuralgic headache. Both constantly happen to the same individuals at different times, and one variety is succeeded by the other. Severe neuralgic headache is often the outcome of the nervous type, and, therefore, we should avoid falling into the error of regarding them as essentially different. They belong to the same great family of neurosal disturbances in the brain, they are intimately related to each other, and they only vary as do the features and mental prociivities of individuals springing from a common parentage.

There are seldom two cases of neuralgic headache precisely alike, either in locality of nerve-disturbance, or in severity; but, making allowance for age, sex, and psychological peculiarities, they have many points in common, and wonderfully harmonise in the several phenomena they induce, when the same set of nerves are involved, and one portion of the brain is congested or hyperæmic - which probably happens, if we may judge of the heat on one side of the head, the flushed cheek, and 
red eye, compared with the normal state of the corresponding side. Still, I would have it understood, that one side of the head may be severely affected when there is extreme pallor of the whole countenance, and, except from the patient's evidence; it would be impossible to say what nerve, or set of nerves are implicated. Minute discrepancies exist in the symptoms experienced by patients of their sufferings from neuralgic or one-sided headache, but the general details are essentially tine same.

Neuralgic Headache (Hemicrania : Migraine of the French, Migrane of the Germans), is one of the most characteristic forms. It possesses a peculiar degree of interest to the physician, and demands a special line of treatment. It may be independent of decayed teeth, malarial poisoning, ovarian excitement, or losses of blood. It may be the simple expression of nerve-exhaustion. With some persons it begins with a slight aching or uneasiness in the superficial branches of the fifth nerve, as they radiate over the temporal region; it is evanescent in itsduration, and interferes but slightly with their duties or pleasures. You would scarcely know they were suffering, if they did not say so ; a mere reticence and dulness of manner may be only observable at first ; but later on, irritability, fretfulness, and disquietude are prominent features of the pronounced condition. They accomplish their duties after a fashion, and the pain is not severe enough to entirely prostrate their energies. And this may be the order of symptoms in a large number of cases-modified neuralgic distress and annoyance destroying all enjoyment while they last, yet never culminating in severe pain. In other instances, and they are invariably the most severe, neuralgic headache is the consequence of the genuine nervous headache attacking the anterior lobe of the brain, or the origin of the fifth nerve, and producing that fearfully distressing pain which compels these patients to isolate themselves from others, and to be alone. Irritability has now vanished, and helplessness and despair take its place.

Neuralgic headache, so resulting, is extremely severe; it induces a great degree of exhaustion, and incapacity for any kind of exertion, mental or bodily. The extremities are cold, the pulse is slow and feeble, the eyes are dull and fixed in the orbits, the pupils are contracted, the eyelids dark and stiff, and the sight is easily wearied. The drawn features, the pallid cheek, the beseeching attitude of the patients as they pace the room, and throw their heads languidly upwards and downwards, tell a tale of indescribable agony, bringing them to the verge of distraction. I have known the collapse so profound when the pain has continued for many hours, that the patient has been almost pulseless, and incapable of swallowing. When at length the seizure subsides, the prostrating effects may be felt for a week afterwards; and some patients complain that their legs are so unsteady that they are unable to walk. These neuralgic headaches are very variable in the way of their onset. A woman, aged 33, when the attack was on her, had every night at bedtime a feeling like the ticking of a watch as soon as she laid her head on the pillow. It would begin, as neuralgic headache generally does, in one temple, or over one eye. She was kept awake at night from a feeling at the back of the eyes like the weight of a heavy stone, giddiness and confusion of ideas succeeding the next day. This patient, nearly all her life from a child upwards, had been subject to bilious headaches; but, when menstruation came on, they changed their character, sickness was no longer a feature of the case, and they became neuralgic or one-sided headaches. She described them as altogether different. When a woman has been subject to nervous headaches all her life, the attacks frequently become aggravated as menstruation is established. A day or two before each returning catamenial period, the patient begins to flag in her health, she is fidgety, loses her appetite, and is sick; but as soon as the pain has come on she is resigned to her fate, and the excitement passes off; she dreams and can obtain no sound sleep at night. The pain keeps over one eye, or concentrates itself over one temple, then it spreads over the entire parietal bone, and finally through the whole head, settling on the top, causing a sensation like a burning coal. Deafness in ore ear is by no means rare, coming on with the attacks of headache, and ceasing with them. In these cases, menstruation may be perfectly normal, there is no dysmenorrhœa, nor is the drain at all profuse. Where it is, we have the type of symptoms described elsewhere ; the brain, robbed of its arterial supply, is very badly nourished; and the cerebral cells are in a state of great irritation.

A case has been mentioned to me, where a lady, when thirty-three years of age, became the subject of neuralgic headache on the left side of the head. It recurred at irregular intervals, till she reached the age of sixty-four, when she was again free, and remained so till her death, at the advanced age of ninety-two, proving how little such nerve-disturbance influences the duration of life. During the time she suffered from the seizures, the eyelids of the affected side lost their normal activity, and one upper eyelid especially became dark, drooping, and wrinkled. In the course of a few years, the eye of the affected side was closed completely, and the patient had no power to separate the lids. After these seizures, and in proportion to the length of interval from suffering, she was able to exercise some power over the eyelids; but gradually they became paralysed, and remained closed. This was possibly owing to a profound impression on the sympathetic system, affecting the superficial nerve-branches distributed to the eyelids, and had no immediate connection with the nerve issuing direct from the brain, because the pupil was normal in size, and the vision was unaffected.

Neuralgic headache is sometimes associated with neuralgia in other parts of the body. I have met with cases in delicate young women, who have suffered on and off for years with pain in the left side of the thorax and over the cardiac region, lasting for an hour and a half at a time, and coming on in bed when at rest, or just before a meal. I have often known it succeed the ordinary sick-headache of early life. A case came before me where sick-headache was occasional till twelve years of age, and then the patient was free till twenty-four years of age, and she so continued till two years and a half after this. Then pain came on in the left temporal region, of a dull, aching, paroxysmal character; but it did not extend over the head. The pain was increased at the catamenial periods, which were profuse. Attention to the uterine functions, and styptic preparations of iron and ergot, completely cured her ; and, three years later there had been no return. The neuralgia vanished on on an improvement of the general health.

Sometimes the disease is more prevalent in a damp situation than it is in a dry one. I knew a lady in 1879 , who had lived in a wet neighbourhood, and, during her residence there, she had neuralgic headache for many years on the left side. On returning to a part of London where the soil was dry and gravelly, the headache left her altogether. Two of her children suffered from headache; one had both near and long sight; the other had headache, with an affection of the third nerve. Thus, we have a strong family predisposition to nerve-disorder.

Among children, I will describe the case of a boy who suffered from a most singular variety of nervous headache. I have never before met with one at all like it. I would call it neuralgia, because it was so paroxysmal in its seizure, though it differed from its customary tendency, in not confining itself, or at least during some part of its duration, to one half of the head; and, unlike frontal headache generally, there was no nausea or loss of appetite.

A. A. was eleven years and a half old when I saw him on January 3 Ist, I879, for the first time. There were seven other children; all of whom, like the patient, were nervous and excitable. One of them, a girl eight years of age, was suffering from a second attack of severe chorea. The father was nervous, the mother self-possessed and healthy. The patient had had measles, but never scarlet fever. He was gifted with rare intellectual ability, and his love of reading was so great, that his chief enjoyment was to lie on a sofa the greater part of the day, and pore over books, even when suffering from much pain and discomfort in his head. On returning from school, in August 1878, he began to experience severe frontal headache, which came on after breakfast, and obliged him to lie down the rest of the day, unable to do anything. A month later, he lost the sensation of taste and smell. From that time, the headache changed its character, and became the same as it was when he consulted me. The taste and smell returned at Christmas. He had now three separate attacks of headache in the course of the day. The first attack began at $8.40 \mathrm{~A} . \mathrm{M}$., and lasted exactly thirty-five minutes; it was always of the same character, and, as to time, his parents were certain that there was no error about it, for this had been tested over and over again without the patient's knowledge. The second attack began at I2.40 P.M., and its duration was also thirty-five minutes. In all respects, it was like the previous seizure. The third attack began at I.40 P.M., and lasted precisely twenty-five minutes. During the seizure, he exhibited the appearance of a person suffering from acute pain. His eyelids were screwed up so as to exclude the light from his eyes; yet vision was not affected. The pain resembled a heavy weight across the whole forehead; he was restless and unable to remain quiet. The tongue was clean, the pulse quiet, and the bowels regular; the urine was pale, acid, and non-albuminous. Many of his teeth were decayed. Several medical men had been consulted, and large doses of quinine, arsenic, croton-chloral, gelseminum, bromides, and bark had been given without avail. One eminent authority thought there might be inflammation in the course of the nerve-trunks. I advised that all study, however light, should be abandoned at once; that he should go to the seaside, and be as much as possible in the open air; that the seizures were to be disregarded, beyond applying hot water to the feet and a mustard leaf to the back of the neck when the attack threatened. His diet was to be plain and nutritious, without a stimulant of any kind. In the shape of medicine, he was to take one- 
thirtieth of a grain of phosphorus in a capsule daily after food for a week, and then to exchange it for a mixture of bromide of potassium and nux vomica for another week. On February 18th, it was reported that he had taken twelve capsules, and a few doses of the mixture. There was only this to remark in the way of change, but at least it was satisfactory - the first morning seizure had in no way changed either in time or in character, but the second now began at II.IO, and the third at I2. IO. They continued as long, and were equally intense. The former medicines were now given up, and he was ordered a drachm of the tincture of quinine in a small cup of milk before getting up in the morning, and a mixture twice a day after food, containing the syrup of the hypophosphite of iron, and bromide of potassium. On March roth, it was reported that he had an excellent appetite, had gained flesh, and seemed well in health. The headaches were the same, except that one day they came on four times in the twenty-four hours, at irregular times, and of varying duration, one lasting only three minutes. $\mathrm{He}$ was advised to go on in the same way, and to be kept apart from the choreic sister. On March 2ist, the report reached me that he was quite well, the headaches having left entirely; and for the previous five days he had been quite free. On the I4th, he had had three attacks as usual, the last being very brief in duration; two on the 15 th, the second being very short; and one on the I6th, which only lasted ten minutes. The medicine was ordered to be continued. On June Ist, his mother wrote to say that he had returned to school a month previously in good health, and that he had now only a headache of an ordinary kind.

This is a rare and curious case, and yet not so curious when carefully inquired into, if we suppose the disease to have been neuralgic, and therefore marked by periodicity ; but it was singular, inasmuch as frontal headache is rarely periodical; it commences in the morning, and usually lasts through the day. A night's rest is generally necessary before relief can be obtained.

This patient returned to school in May and remained till August, when he only suffered from an occasional headache, and that chiefly at breakfast-time. It went off during the day, but it sometimes recurred during any excitement. There was no sickness with it. He described this headache as being quite different from the former seizures. He was now restless, moving from one chair to another, unable to remain quie for an instant; his appetite was capricious, and his heart agitated. Bromide of ammonium and the ammonio-citrate of iron with strychnia were ordered to be taken twice a day after food. He went to the seaside.

I will now consider another curious type of neuralgic headache, not so much in the character of the pain and its situation as in the consequences resulting from it, and the altered appearance of the patient's features.

Miss B., aged 29, of dark hair and complexion, consulted me on March 18th, 1879, for neuralgic headache, which she traced to anxiety. It affected her left temple, left brow, extending round the eye, and down the left side of the body. She was stout and healthy looking, and had never suffered any definite illness. The pain was described as dull and heavy; it begins on waking, and goes off at mid-day. It did not prevent her from following her occupation. The neuralgic headache was attended with a burning sensation of the face, which was flushed and hot, and it continued for fifteen minutes. This variety of headache never came on till she suffered much worry of mind. She was now irritable and easily put out, which was not usual with her. The pulse was quiet, the tongue clean, the bowels and catamenia regular. The heart's sounds were normal, and the urine was nonalbuminous. In addition to this kind of headache, she experienced one of quite a different character, which she called bilious; she had had it at intervals for eighteen months; when the attack was on her, she became pale and vomited, and her skin was cold and shrivelled. But the singular part of the case is, that the eyelashes of the upper and lower left eyelid had been dropping out during the last two months, and the chief of those that remained (about seventy) were perfectly white. The eyelid was thickened and inflamed. She thought the change was quicker in the lower than in the upper eyelid, and she did not observe it till the change took place. The left eytlid drooped a little more than the right, and it was always worse if she were fatigued or worried, and especially when the headache was bad. She complained, too, of some dimness of vision, and that the eye itched; but she saw well at the ordinary visual distance. There were also patches of pityriasis over the left cheek and half the lip, where the hairs were also turned white. - Over the scalp on the affected side were spots of alo-

* This is a point of great clinical importance, to which $I$ shall allude again when I speak of bilious or sick headache. Sufferers from nervous or neuralgic headache often tell me that they get once or twice a year a common or bilious headache as they term it: it affects the whole forehead and vertex; the pain is acute an throbbing, as if the brain would burst; and there is fearful nausea and vomiting. pecia, but there was no change in the colour of the skin on the right side of the face.

Mr. W. H. Power, who made an ophthalmoscopic examination, reported that "the discs of, both eyes can be very well seen through the perfectly clear media. They present a very well-defined central (physiological) depression, the borders of which are unusually steep. The rest of the disc is of a normal colour, with clean cut periphery. The vessels are unusually large and numerous. The rest of the fundus, fovea centralis, and adjoining parts appear to be healthy". There was an error of refraction in both eyes, but particularly the left. Quinine and arsenic, with bromide of potassium, were prescribed, and an ointment of vaseline and binoxide of mercury was ordered to be applied to the offending cilia.

In women who have been for some years sufferers from nervous headache, whether neuralgic or not, we may sometimes notice yellow patches, having the tint of chamois leather, looking like small ruptured encysted tumours, containing caseous matter, lying superficially beneath the skin. The patches are soft, irregular in outline, and may remain stationary for years without increasing in size. There is no tendency to ulceration or suppuration. They are generally seen on the upper and lower eyelids, at the inner canthus, and the patches are permanent. The affection is seen in cases of severe nervous headache, which have lasted for years, and they always attest the severity of the paroxysms. I have not noticed these spots in young subjects. They all occurred in women of middle or advanced life, and several had experienced uterine disorder, especially menorrhagia. In two well-marked cases, one was the subject of hepatic congestion, and the other died of malignant disease of the liver. Not that I believe for a moment there is any relation between these spots and liver-disease, seeing that these peculiar spots are almost exclusively confined to women. This complaint is the Xanthelasma Palpebrarum, described by Erasmus Wilson, Hutchinson, Liveing, Tilbury Fox, and other dermatologists. Hutchinson, who has studied this curious complication very minutely, has found it occurring in association with any disorder that can produce dark areolæ around the eyes. "The minute anatomy of xanthoma was first accurately described by Waldeyer and Murchison; it consists of a fibro-cellular growth, infiltrated with a yellowish oil, which is found in and around the cells; it is this oily fat which gives to the structure its bright yellow colour, and it is well to remember that it is an essential part of the disease, and not the result of degeneration" (Handbook of the Diagnosis of Skin-Diseases, by R. Liveing, M.D., page 209).

3. Occipital Headache. - This is another variety of nervous headache, which possesses much interest to the physician. It is so intimately connected with nervous and neuralgic headache, as to justify a separate classification. It is a form of headache which would be shrouded in mystery, if the anatomical arrangement of the implicated nerves were not well understood. The free communication between the pneumogastric nerve and the inferior cervical ganglion of the sympathetic (which supplies a plexus to the vertebral artery), readily effects a trans. ference of morbid impressions received by the peripheral extremities of branches of the vagus in the abdominal or thoracic viscera to the vasomotor nerves of the chief nutrient vessels of the occipital region. In a similar manner, the vaso-motor nerves of the occipital and posterior auricular arteries may be affected.* In fact, if looked at through the wide area of nerve-communication, it will explain the gastric symptoms, the sickness, the nausea, and vomiting, when the great sympathetic and par vagum are in unfriendly union. Again, congestion of the lateral and occipital sinuses is a most probable occurrence in languid individuals, who continue in a recumbent posture for a longer period than is required for necessary repose. Headache, produced by such congestion, is the homologue of the hypostatic pneumonia of patients kept for a long period in the supine position, during recovery from surgical injuries and operations. Lastly, rheumatism may happen to attack the pericranium or dura mater in the occiput. But, when we have to deal with occipital headache, the possibility of disease of the occipito-atlantoid or atlo-axial articulations must never be overlooked when the pain is fixed in this situation, and rotation of the neck is painful. I have elsewhere referred to cases, in corroboration of Mr. Hilton's statement that pain at the back of the head is sometimes owing to organic disease (On Headaches, third edition, I880, page 104). A patient, who was in the last stage of consumption, came under my care in 1869 . He had great pain over the occipital region, and he could not move his head for weeks together. It was pitiable to watch the expression of his countenance when heswallowed food, or when an attempt was made to move him from one side of the bed to the other. Two

For an account of the relation of innervation to pain in the occiput, see "The Etiology and Treatment of Occipital Headache", by Dr. Woakes (Practitioner, vol. $x x, p, 263)$. 
months before his death the pain abated, and he found he could hear a grating noise in the neck on slight rotation of the head. On a post mortem examination, the two first cervical vertebræ were found to be carious.

Mr. Hilton has recorded the case of a gentleman whom he saw in consultation with Dr. Bright. The patient was twenty-eight years of age, and had suffered from pain at the back of the head for some time. It was thought to be rheumatic. "The cause of the pain was the question to be decided. Dr. Bright entertained an opinion that pain at the back of the head resulted somefimes from the close proximity of the vertebral artery to the suboccipital nerve, between the occiput and the atlas, or to the great occipital nerve, between the atlas and axis, and that the pressure of the artery upon one of these nerves produced the pain at the back of the head; and herein is the chief reason for my mentioning this individual case, because I believe the explanation will not hold good as regards the suboccipital nerve, for that nerve rarely sends normally any filaments to the skin beyond the muscles; indeed, it is chiefly a motor nerve coming off only from the anterior part of the spinal marrow. The great occipital nerve supplied the skin where the pain was felt by this gentleman, and in tracing this nerve towards the spine we came to the cause, viz., disease between the first and second cervical vertebræ. The patient was ultimately cured by continued rest in the nearly horizontal position; the cure, however, consisted in complete ankylosis of the bones, and a fixed neck, with the head turned somewhat downwards and towards the left side. This patient died from pulmonary consumption twelve years after his recovery from his disease of the spine" (On Pain and the Therapeutic Influence of RestThe Lancet, vol. ii, 1860, page 379).

I have noticed occipital pain often enough in cases of nervous and neuralgic headache. When the patient has a mere tingling sensation at the top and side of the head; the pain at the back of the neck may be almost unendurable; it may fix itself over the occipital protuberance and involve the lower half of the bone from ear to ear, so that he feels he canniot support his head. If the pulse be slow and feeble, and there are the symptoms of acute congestion, the cerebellum and the veins in its neighbourhood are probably so full of blood as to act prejudicially on some part of the brain itself.

Professor Hermann Meyer states that the right lateral sinus is larger than the left, and that the blood which flows through it goes on in a quicker stream than that which passes through the left side. This, he says, is owing to the more vertical course of the right innominate vein, which is almost in a straight line with the vena cava superior below it, and with the internal jugular beyond it, the sinus opening directly into the latter vein. The more horizontal position of the left innominate vein probably retards the return of blood from the left lateral sinus (the London Medical Record, October 15th, 1878, page 431).

From all that has been said, we shall scarcely overlook the importance of carefully inquiring whether the patient who complains of occipital headache has at any time received a blow at the back of the head, which might be forgotten with the lapse of time. Slight injuries received in early life at the back of the neck, over the spine, have been followed in after years by disease of the vertebre and the brain itself. Dr. Bastian has recorded two cases of children, aged respectively five and ten, where symptoms, indicative of disease of the cerebellum, followed an injury to the occiput. Dulness of manner, attacks of vertigo, sickness, staggering gait, and severe occipital pains, lasting many months, were the leading symptoms in one case. An improvement took place under iodide of potassium and cod-liver oil. In the second case, the occipital pains were associated with vomiting, and, after the patient had remained in bed five weeks, "she had a sort of fit". She could not support her head, and staggered in her walk. Her sight also began to fail. Similar treatment was adopted to that of the first case, and a year later she was well, except that in walking she kept her legs apart, and slightly reeled (Ibid., page 436). In the first case, Dr. Bastian thinks it not improbable that a small tumour may have formed, and that, in the second, there was a small abscess which had become absorbed, and the cyst shrunken. It would seem to me that, in the absence of fever, epileptic seizures, or rigors, severe congestion or slight inflammatory effusion would be enough to account for the symptoms in each case.

I will consider on a future occasion those varieties of nervous headache attended with a disturbance of vision, and with disordered sensation of the upper and lower extremities.

Glasgow Aberdeenshire Association Medical Bursary.This bursary, of the annual value of fifteen guineas, is tenable for three years by a medical student of the University of Aberdeen. After competitive examination, it has been awarded to Mr. John Gordon, of Aberdeen.

\section{THE TREATMENT OF RINGWORM BY CROTON OIL.}

By ROBERT LIVEING, M.D., F.R.C.P.,

Physician to the Department for Diseases of the Skin at Middlesex Hospital.

THERE is no lack of efficient remedies for common ringworm; sulphur, salts of mercury, carbolic acid, chrysophanic acid, iodine, and many more; but there is one essential condition of success in treatment on which everything, as it were, depends, and that is, that the remedy be brought into close contact with the disease; without this, all remedies are equally useless. In illustration of this point, we constantly see a patch of ringworm on the neck cured by a single application of iodine ; but it may take years to cure a similar patch on the scalp. The disease is exactly the same in the two cases, and would be just as easily cured in the one as in the other, if only the remedy could be brought into direct contact with the parasites, as indeed readily happens in ringworm of the scalp in infants. The proverb, "You may bring a horse to the water, but you cannot make him drink," is, as it were, reversed in ringworm, for if you can only get him to the water he is bound to drink; the difficulty is getting him there. Now, croton-oil supplies us with just such a remedy as we want, for it has the property of reaching the disease, and setting up a deep seated follicular inflammation which destroys the trichophyton. Although this remedy has long been used in France, we are indebted to Dr. Alder Smith for thoroughly working out the subject in this country ; and more especially for pointing out clearly the object to be aimed at, namely, the artificial production of kerion over a small surface. Croton-oil liniment is very useful as an irritant, just as iodine liniment is ; but, for the special purpose of setting up a kerion, or deep-seated inflammation of the follicles, pure croton-oil is best. It is now about four years since Dr. Alder Smith called my attention to the subject; and since that time I have adopted the croton-oil treatment in a fair number of carefully chosen cases, and always hitherto with satisfactory results. I would add, however, that it is quite possible to produce a slough, and consequently a permanent bald patch, by the too free use of croton-oil, just as sometimes happens accidentally from the application of other strong remedies in more common use; and further, if croton-oil be used without discrimination, or a due regard to the age of the patient and the extent of the surface involved, it is sure to be brought into unmerited disrepute, the fault being, not in the croton-oil, but in the person who recommended it in unsuitable cases.

I can best enforce this by an example, thus : a typical case for the use of croton-oil, and one which frequently occurs in practice, is that of a boy aged from twelve to fourteen years (when time becomes valuable), who is very anxious to return to school, but is prevented by one or two spots of intractable ringworm. In this case, and in others like it, croton-oil is invaluable, and the little extra trouble it gives is readily borne for the sake of saving perhaps a year at school.

The best way of using the croton-oil is as follows. A single small spot, not larger than a shilling, should be treated first; the croton-oil should be carefully but thoroughly painted on with a small stiff camel's hair brush ; a few hours afterwards, a warm poultice should be applied and kept on all night. The croton-oil must usually be applied again the next day, followed as before by constant poulticing; a third and sometimes a fourth application may be necessary to set up the required amount of inflammation; the skin should be swollen, boggy, and discharging freely, like a natural kerion ; the production of this is much favoured by warm fomentions and poultices. As soon as this condition is produced, no more croton-oil should be applied; frequent warm fomentation is all that is then necessary ; and when, in the course of a day or two, the inflammation has a little subsided, the stumpy hairs should be extracted entire ; this is easily done, as they are for the most part loose in their follicles, and can then be removed without pain. If a good kerion have been established, the cure is complete; if only an imperfect one have been formed the process may, after some time, have to be repeated over part of the same area. It is astonishing how quickly the new hair generally grows over the bald patch when the cure is complete. It is true that natural kerion is sometimes, though rarely, followed by a permanent bald spot, and it is conceivable that such an accident might follow from the use of croton-oil, without any error in the choice of the case or in the application of the remedy; I would add, however, that I have never yet met with such a result ; if it occurred at all frequently, we might perhaps turn croton-oil to account in the removal of superfluous hair.

Those who wish to use croton-oil with safety and success should adopt the following rules, which err, perhaps, a little on the side of caution : -1. Not to use it for children under ten years of age; 2 . Never to apply it at any one time to a surface larger than a florin; 3 . To use it 